\title{
Analytical Method for Solving the Fractional Order Generalized KdV Equation by a Beta-Fractional Derivative
}

\author{
Majid Bagheri $(\mathbb{D})$ and Ali Khani $(\mathbb{D}$ \\ Faculty of Science, Department of Applied Mathematics, Azarbaijan Shahid Madani University, Tabriz, Iran \\ Correspondence should be addressed to Ali Khani; khani@azaruniv.ac.ir
}

Received 14 August 2020; Revised 27 September 2020; Accepted 11 October 2020; Published 4 November 2020

Academic Editor: Soheil Salahshour

Copyright @ 2020 Majid Bagheri and Ali Khani. This is an open access article distributed under the Creative Commons Attribution License, which permits unrestricted use, distribution, and reproduction in any medium, provided the original work is properly cited.

\begin{abstract}
The present work is related to solving the fractional generalized Korteweg-de Vries (gKdV) equation in fractional time derivative form of order $\alpha$. Some exact solutions of the fractional-order gKdV equation are attained by employing the new powerful expansion approach by using the beta-fractional derivative which is used to get many solitary wave solutions by changing various parameters. The obtained solutions include three classes of soliton wave solutions in terms of hyperbolic function, trigonometric function, and rational function solutions. The obtained solutions and the exact solutions are shown graphically, highlighting the effects of nonlinearity. Some of the nonlinear equations arise in fluid dynamics and nonlinear phenomena.
\end{abstract}

\section{Introduction}

The differential equation of fractional order is the new form of classical integer order differential equations. Different types of differential equations of both ordinary differential equations (ODEs) and partial differential equations (PDEs) in various fields of science like fluid mechanics and biological systems are expressed in fractional forms [1]. There is no any particular method for accessing the exact type of solutions of fractional PDEs, but some approximate solutions are determined by using the Adomian decomposition approach, the homotopy perturbation approach, and the homotopy analysis approach [2-4]. The analytical method and its various forms are well known for determining soliton wave-type solutions of the nonlinear PDEs. With chronology, some investigators have utilized the new analytical approach on fractional-type nonlinear PDEs for obtaining the solitary solutions. This work is related to the fractional order generalized Korteweg-de Vries (gKdV) equation [5]; for this, the fractional form of the gKdV equation [5] is taken as

$u_{t}^{\alpha}+F(u) u_{x}+u_{x x x}=0, \quad 0 \leq \alpha<1,0<d<4, F(u)=\lambda u^{d}+\mu u^{2 d}, \lambda, \mu \in R$.
Equation (1) is a fractional form of the classical generalized Korteweg-de Vries equation which exists by changing the 1st-order time derivatives by fractional derivatives.

In the past two decades, the fractional calculus theory gained great attention and popularity in various fields of science and engineering due to its demonstrated applications. These contributions to the fields of sciences and engineering are based on mathematical analysis. They cover widely known classical fields such as Abel's integral equation and viscoelasticity. They also include the analysis of feedback amplifiers, the fractional-order Chua-Hartley systems, electrode-electrolyte interface models, fractionalorder models of neurons, electric conductance of biological systems, generalized voltage dividers, fitting of experimental data, capacitor theory, and the fields of special functions [6-9].

Several robust methods have been used to solve the FDEs, the fractional differential equations and dynamic systems containing fractional derivatives. Some of the most important methods are Adomian's decomposition method [1012], the exp-function method [13], He's variational iteration method [14, 15], the fractional subequation method [16], the first integral method [17], the homotopy analysis method [18], the $\left(G^{\prime} / G\right)$-expansion method [19], the homotopy 
perturbation method [20,21], the spectral methods [22], and the transform methods [23]. In [24], the authors presented two methods, which are the $\exp (-\phi(\xi))$-expansion method and the Kudryashov method. In [25], the authors demonstrated three methods, which are the csch function method, the tanh-coth method, and the modified simple equation method. In [26-29], the authors introduced the semiinverse variational principle method, the extended Kudryashov method, the modified simple equation method, and the expanded trail equation method, respectively. Moreover, the fractional differential equations have been studied by powerful authors, and their applications were introduced in sciences and engineering branches [30-32].

One of the well-known equations is the ZK equation, first obtained as a description of weakly nonlinear ion-acoustic modes in a strongly magnetized plasma; it is of particular interest as it is the simplest equation that admits cylindrical and spherical solitary wave solutions in addition to the planar $\mathrm{KdV}$ soliton solutions [33]. Another powerful analytical method is called the exp-function method (EFM), which was first presented by He [34]. EFM has successfully been applied to many situations. For example, He and Wu [34] solved the nonlinear wave equations via EFM. Abdou [35] solved generalized solitonary and periodic solutions for nonlinear partial differential equations by EFM. For further information, refer to vigorous references in ([35-40]). In the following years, this proposed method was improved by many researchers. Yang developed general fractional derivatives, along with theory, methods, and applications, to some nonlinear fractional differential equations [41]. Recently, there has been a new fractal nonlinear Burgers' equation arising from the acoustic signal propagation studied by Yang [42]. Also, Yang et al. investigated fundamental solutions of anomalous equations implemented with the decay exponential kernel [43]. A new integral transform operator for solving the heat-diffusion problem has been utilized by Yang [44]. In [45], Liu et al. probed the group analysis to the time fractional nonlinear wave equation and found many exact solutions. Moreover, Liu et al. worked on the time-fractional nonlinear diffusion equation [46]. Same authors proposed the fractional symmetry group method for the time fractional nonlinear heat conduction equation which usually appears in mathematics, physics, integrable systems, fluid mechanics, and nonlinear areas [47]. Furthermore, in the last decade, many real world problems have been explained using fractional partial differential equations which can be linked to the following valuable researches: utilizing the $q$-homotopy analysis transform method and the fractional natural decomposition method for the fractional Benney-Lin equation [48]; applying the quasiaffine biframelets to the fractional differential equations [49]; analyzing the moderate epidemiological model to describe computer viruses with an arbitrary order derivative [50]; solving the fractional Kaup-Kupershmidt equation with the Atangana-Beleanu derivative and the Caputo-Fabrizio derivative [51]; employing the RiemannLiouville integral, the Atangana-Baleanu integral operator, and the nonlinear Telegraph equation [52]; solving singular fractional integrodifferential equations with applications to hematopoietic stem cell modeling [53]; applying the truncated $M$-fractional derivative to fractional differential equations [54]; applying the $\left(m+1 / G^{\prime}\right)$-expansion method to the study of the $(2+1)$-dimensional hyperbolic nonlinear Schrödinger equation [55], the study of unreported cases of 2019-nCOV epidemic outbreaks [56-58], and the study of the conformable $(2+1)$-dimensional Ablowitz-Kaup-NewellSegur equation [59]; and employing a reliable hybrid numerical method for a time-dependent vibration model [60]. To make this paper more self-contained, a general trigonometric, hyperbolic, and exponential function of the $\mathrm{gKdV}$ equation is constructed with the help of an expansion approach, which is crucial in obtaining the lump solution of equation (1).

The pattern of this article is summarized as follows: Section 2 gives the details of the initial definitions; Section 3 gives an introduction of the direct truncation method which is utilized for getting the exact solutions of the gKdV equation in Section 4; Section 5 gives the numerical simulation and graph details; and Section 6 gives some conclusions at the end.

\section{Initial Definitions}

Definition 1. Definition of $\beta$-derivative: suppose $\varphi:[0 ; 1)$

$\rightarrow \mathbf{R}$, then the $\beta$ derivative of $\varphi$ of order $\alpha$ is defined as

$D_{t}^{\alpha}(\varphi)(t)=\lim _{\varepsilon \rightarrow 0} \frac{\varphi\left[t+\varepsilon(t+1 / \Gamma(\alpha))^{1-\alpha}\right]-\varphi(t)}{\varepsilon}, \quad \alpha \in(0,1], t>0$.

The following properties and new theorems will be used.

Theorem 2. Suppose $\alpha \in(0,1]$; let $\varphi$, $\omega$ be $\alpha$-differentiable at point $t$. Therefore, we have

$$
\begin{aligned}
D_{t}^{\alpha}(a \varphi(t)+b \omega(t)) & =a D_{t}^{\alpha}(\varphi(t))+b D_{t}^{\alpha}(\omega(t)), \quad \text { for } a, b \in \mathbf{R}, \\
D_{t}^{\alpha}(c) & =0, \quad \text { for } c \in \mathbf{R}, \\
D_{t}^{\alpha}(\varphi(t) \omega(t)) & =\varphi(t) D_{t}^{\alpha}(\omega(t))+\omega(t) D_{t}^{\alpha}(\varphi(t)), \\
D_{t}^{\alpha}\left(\frac{\varphi(t)}{\omega(t)}\right) & =\frac{\varphi(t) D_{t}^{\alpha}(\omega(t))-\omega(t) D_{t}^{\alpha}(\varphi(t))}{\omega^{2}(t)}, \\
D_{t}^{\alpha} \varphi(t) & =\left(t+\frac{1}{\Gamma(\alpha)}\right)^{1-\alpha} \frac{d \varphi(t)}{d t} .
\end{aligned}
$$

Theorem 3 (see [61-63]). Suppose $\varphi:[0 ; 1) \rightarrow \mathbf{R}$ is a function such that $\varphi$ is differentiable and also $\alpha$-differentiable. Assume $\omega$ is a differentiable function defined in the range of $\varphi$. Therefore, we have

$$
D_{t}^{\alpha}(\varphi \circ \omega)(t)=\left(t+\frac{1}{\Gamma(\alpha)}\right)^{1-\alpha} \omega^{\prime}(t) \chi^{\prime}(\omega(t))
$$

where a prime denotes the classical derivatives with respect to $t$. 


\section{Methodology}

In this section, we give a description of the direct truncation method and introduce it to a partial differential equation.

For a given partial differential equation

$$
P\left(u, u_{x}, u_{x x}, \cdots, D_{t}^{\alpha} u, D_{x}^{\alpha} u, D_{x x}^{\alpha} u, \cdots\right)=0, \quad 0<\alpha \leq 1 .
$$

Using a transformation as

$$
\begin{aligned}
u(x, t) & =u(\phi), \\
\eta & =k x+\frac{l}{\alpha}\left(t+\frac{1}{\Gamma(\alpha)}\right)^{\alpha},
\end{aligned}
$$

where $k$ and $l$ are constants to be determined later. We can rewrite equation (5) in the following nonlinear ODE:

$$
Q\left(u, k u^{\prime}, k^{2} u^{\prime \prime}, \cdots, l u^{\prime}, \cdots\right)=0
$$

where the prime denotes the derivative with respect to $\phi$. If possible, we integrate equation (7) term by term one or more times. This yields constants of integration. For simplicity, the integration constants can be set to zero. Suppose $g$ has the following truncation form:

$$
g(\phi)=\frac{\sum_{j=0}^{\tau} a_{j} \xi(\phi)^{j}}{\zeta(\phi)^{\tau}}
$$

in which $\xi(\phi)$ and $\zeta(\phi)$ are introduced in the following forms:

$$
\begin{aligned}
& \xi(\phi)=p_{1} F(\chi(\phi))+q_{1} G(\chi(\phi))+r_{1}, \\
& \zeta(\phi)=p_{2} F(\chi(\phi))+q_{2} G(\chi(\phi))+r_{2}, \\
& u(\xi)=g(\phi)=\frac{\sum_{j=0}^{\tau} a_{j}\left(p_{1} F(\chi(\phi))+q_{1} G(\chi(\phi))+r_{1}\right)^{j}}{\left(p_{2} F(\chi(\phi))+q_{2} G(\chi(\phi))+r_{2}\right)^{\tau}},
\end{aligned}
$$

where $a_{j}, p_{1}, q_{1}, r_{1}, p_{2}, q_{2}$, and $r_{2}$ are constants to be determined, $\chi(\phi)$ is given, and eitherFandGare functions determined by an ordinary differential system or $F$ and $G$ are functions given by direct ansatz such that their derivations are combinations of $F$ and $G$, and $\chi(\phi)$ is determined by an ordinary differential equation:

$$
\frac{d \chi(\phi)}{d \phi}=H(\chi(\phi))=L F(\chi(\phi))+M G(\chi(\phi))+N
$$

in which the function $H$ is also given by a direct ansatz according to the context, and the exponent $\tau$ is determined by utilizing the homogeneous balance method in equation (5). The value $\tau$ is determined by equalizing the maximum order nonlinear term and the maximum order partial derivative term appearing in (7). If $\tau$ is rational, then the appropriate transformations can be applied to conquer these hurdles. Substituting (9) and (10) into (8) leads to a polynomial in $F(\phi)$ and $G(\phi)$; then, we set the coefficients of $F^{i}(\phi) G^{j}(\phi)$ and the constant term to zero to get a system of algebraic equations on the unknown parameters in $H$ together with the unknown num- bers $a_{j}, p_{1}, q_{1}, r_{1}, p_{2}, q_{2}$, and $r_{2}$ for $j=0,1, \cdots, \tau$. By solving the system, one can get $a_{j}, p_{1}, q_{1}, r_{1}, p_{2}, q_{2}$, and $r_{2}$ and the unknown parameters in $H$; then, by solving equation (10) to get $\chi(\phi)$, the solutions of equation (5) can be obtained.

\section{Traveling Wave Solution Fractional Order Form of Generalized KdV Equation}

The given section deals with the application of a new powerful expansion technique by determining the traveling wave form solutions of the fractional order generalized $\mathrm{KdV}$ equation:

$$
u_{t}^{\alpha}+F(u) u_{x}+u_{x x x}=0, \quad F(u)=\lambda u^{d}+\mu u^{2 d}, \lambda, \mu \neq 0,
$$

by applying the aforementioned method. By using the fractional beta complex transform $\eta=k x+(l / \alpha)(t+(1 / \Gamma(\alpha)))^{\alpha}$, equation (11) is reduced to the following ODE:

$$
l u^{\prime}+\left(\lambda u^{d}+\mu u^{2 d}\right) k u^{\prime}+k^{3} u^{\prime \prime \prime}=0
$$

Integrating equation (12) once and setting the constant of integration equal to zero results in

$$
l u+\frac{k \lambda}{d+1} u^{d+1}+\frac{k \mu}{d+1} u^{2 d+1}+k^{3} u^{\prime \prime}=0 .
$$

Balancing $u^{\prime \prime}$ and $u^{2 d+1}$ by employing the homogenous principle, we get

$$
M+2=(2 d+1) M, \quad \Rightarrow M=\frac{1}{d} .
$$

To get a closed form solution, we use the transformation

$$
u(\eta)=v(\eta)^{(1 / d)}
$$

Substituting (15) into equation (13), we get

$l v^{2}+\frac{k \lambda}{d+1} v^{3}+\frac{k \mu}{2 d+1} v^{4}+\frac{k^{3}}{d}\left(\frac{1-d}{d} v^{\prime 2}+v v^{\prime \prime}\right)=0$

Balancing $v v^{\prime \prime}$ and $v^{4}$, we get

$$
2 M+2=4 M, \quad \Rightarrow M=1 .
$$

Case I. Then, the exact solution will be as

$$
v(\eta)=\frac{e^{2 \chi(\phi)} a_{1} p_{1}+e^{\chi(\phi)} a_{1} q_{1}+a_{1} r_{1}+a_{0}}{p_{2} e^{2 \chi(\phi)}+q_{2} e^{\chi(\phi)}+r_{2}} .
$$

Inserting (18) into equation (16), we obtain the below relation 


$$
\begin{aligned}
& \left(d^{2}(d+1)(2 d+1)\left(p_{2} e^{2 \chi(\phi)}+q_{2} e^{\chi(\phi)}+r_{2}\right)^{4}\right)^{-1} \sum_{n=0}^{11} C_{n} \exp \\
& \cdot(n \chi(\phi))=0,
\end{aligned}
$$

where $C_{n}(0 \leq n \leq 11)$ are polynomial statements in terms of $a_{0}, a_{1}, p_{1}, p_{2}, q_{1}, q_{2}, r_{1}$, and $r_{2}$. Hence, solving the resulting system $C_{n}=0(0 \leq n \leq 11)$ simultaneously, we acquire the following sets of parameters of solutions.

Set $I$.

$$
\begin{aligned}
L & =0, \\
M & =0 \\
N & =\frac{1}{2} \frac{\sqrt{-\mu(d+1)(2 d+1)} \lambda d}{\mu(d+1)(d+2) k} \\
k & =k, \\
l & =\frac{\lambda^{2}(2 d+1) k}{\mu\left(d^{3}+5 d^{2}+8 d+4\right)}, \\
a_{0} & =-\frac{r_{2} \lambda(2 d+1)}{(d+2) \mu}, \\
a_{1} & =0, \\
p_{1} & =p_{1}, \\
p_{2} & =p_{2} \\
q_{1} & =q_{1} \\
q_{2} & =0 \\
r_{1} & =r_{1}, \\
r_{2} & =r_{2} .
\end{aligned}
$$

We, therefore, gained the following generalized solitary solution:

$$
\begin{aligned}
& u_{1}(\phi)=\left(-\frac{r_{2} \lambda(2 d+1)}{(d+2) \mu\left(p_{2} e^{2 \chi(\phi)}+r_{2}\right)}\right)^{(1 / d)}, \\
& \chi(\phi)=\frac{1}{2} \frac{\sqrt{-\mu(d+1)(2 d+1)} \lambda d}{\mu(d+1)(d+2) k}(\phi+C),
\end{aligned}
$$

in which

$$
\phi=k x+\frac{\lambda^{2}(2 d+1) k}{\mu \alpha\left(d^{3}+5 d^{2}+8 d+4\right)}\left(t+\frac{1}{\Gamma(\alpha)}\right)^{\alpha} .
$$

Set II.

$$
\begin{aligned}
L & =0, \\
M & =0, \\
N & =\frac{\sqrt{-\mu(d+1)(2 d+1)} \lambda d}{\mu(d+1)(d+2) k}, \\
k & =k, \\
l & =\frac{\lambda^{2}(2 d+1) k}{\mu\left(d^{3}+5 d^{2}+8 d+4\right)}, \\
a_{0} & =-\frac{r_{2} \lambda(2 d+1)}{(d+2) \mu}, \\
a_{1} & =0, \\
p_{1} & =p_{1}, \\
p_{2} & =0, \\
q_{1} & =q_{1}, \\
q_{2} & =q_{2}, \\
r_{1} & =r_{1}, \\
r_{2} & =r_{2} .
\end{aligned}
$$

We, therefore, gained the following generalized solitary solution:

$$
\begin{aligned}
u_{2}(\phi) & =\left(-\frac{r_{2} \lambda(2 d+1)}{(d+2) \mu\left(q_{2} e^{\chi(\phi)}+r_{2}\right)}\right)^{(1 / d)}, \\
\chi(\phi) & =\frac{\sqrt{-\mu(d+1)(2 d+1)} \lambda d}{\mu(d+1)(d+2) k}(\phi+C),
\end{aligned}
$$

in which

$$
\phi=k x+\frac{\lambda^{2}(2 d+1) k}{\mu \alpha\left(d^{3}+5 d^{2}+8 \quad d+4\right)}\left(t+\frac{1}{\Gamma(\alpha)}\right)^{\alpha} .
$$

Set III.

$$
\begin{aligned}
L & =0, \\
M & =M, \\
N & =N, \\
k & =\frac{\sqrt{-\mu(d+1)(2 d+1)} \lambda d}{\mu(d+1)(d+2) N}, \\
l & =\frac{\lambda^{3}(2 d+1) \sqrt{-\mu(d+1)(2 d+1)}}{\mu^{2}(d+1)\left(d^{3}+5 d^{2}+8 d+4\right)(d+2) N}, \\
a_{0} & =-\frac{\left(M q_{2}-N p_{2}\right) \lambda(2 d+1) r_{1}}{N(d+2) \mu p_{1}}, \\
a_{1} & =\frac{\left(M q_{2}-N p_{2}\right) \lambda(2 d+1) r_{1}}{N(d+2) \mu p_{1}}, \\
p_{1} & =p_{1}, \\
p_{2} & =p_{2}, \\
q_{1} & =0 \\
q_{2} & =q_{2}, \\
r_{1} & =r_{1}, \\
r_{2} & =0 .
\end{aligned}
$$


We, therefore, gained the following generalized solitary solution:

$$
\begin{aligned}
& u_{3}(\phi)=\left(\frac{\left(M q_{2}-N p_{2}\right) \lambda(2 d+1) \mathrm{e}^{\chi(\phi)}}{N(d+2) \mu\left(\mathrm{e}^{\chi(\phi)} p_{2}+q_{2}\right)}\right)^{(1 / d)}, \\
& \chi(\phi)=N(\phi+C)+\ln \left(\frac{N}{1-M \exp (N(\phi+C))}\right),
\end{aligned}
$$

in which

$$
\begin{aligned}
\phi= & \frac{\sqrt{-\mu(d+1)(2 d+1)} \lambda d}{\mu(d+1)(d+2) N} x \\
& +\frac{\lambda^{3}(2 d+1) \sqrt{-\mu(d+1)(2 d+1)} d}{\mu^{2} \alpha(d+1)\left(d^{3}+5 \quad d^{2}+8 \quad d+4\right)(d+2) N} \\
& \cdot\left(t+\frac{1}{\Gamma(\alpha)}\right)^{\alpha} .
\end{aligned}
$$

Set IV.

$$
\begin{aligned}
L & =0, \\
M & =M, \\
N & =\frac{1}{2} \frac{\sqrt{-\mu(d+1)(2 d+1)} \lambda d}{\mu(d+1)(d+2) k}, \\
k & =k \\
l & =\frac{\lambda^{2}(2 d+1) k}{\mu\left(d^{3}+5 d^{2}+8 d+4\right)}, \\
r_{1} & =r_{1}, \\
r_{2} & =r_{2}, \\
a_{0} & =\frac{\left(4 M^{2} d^{3} k^{2} \mu r_{2}+20 M^{2} d^{2} k^{2} \mu r_{2}+32 M^{2} d k^{2} \mu r_{2}+16 M^{2} k^{2} \mu r_{2}+2 d^{3} \lambda^{2} p_{2}+d^{2} \lambda^{2} p_{2}\right) r_{1}}{(d+2) \mu d^{2} \lambda p_{1}} \\
p_{1} & =p_{1}, \\
p_{2} & =p_{2}, \\
q_{1} & =0, \\
a_{1} & =-\frac{4 M^{2} d^{3} k^{2} \mu r_{2}+20 M^{2} d^{2} k^{2} \mu r_{2}+32 M^{2} d k^{2} \mu r_{2}+16 M^{2} k^{2} \mu r_{2}+2 d^{3} \lambda^{2} p_{2}+d^{2} \lambda^{2} p_{2}}{(d+2) \mu d^{2} \lambda p_{1}} \\
q_{2} & =4 \frac{r_{2} M(d+2) k \mu(d+1)}{\sqrt{-\mu(d+1)(2 d+1)} \lambda d} .
\end{aligned}
$$

We, therefore, gained the following generalized solitary solution:

$$
u_{4}(\phi)=-\frac{1}{2} \frac{\left(4 M^{2} k^{2} \mu r_{2}(d+1)(d+2)^{2}+d^{2} \lambda^{2} p_{2}(2 d+1)\right) \sqrt{-\mu(d+1)(2 d+1)} \mathrm{e}^{2 \chi(\phi)}}{\mu^{2}(d+1) d(d+2)\left(1 / 2\left(\sqrt{-\mu(d+1)(2 d+1)} \lambda d\left(p_{2} \mathrm{e}^{2 \chi(\phi)}+r_{2}\right) / \mu(d+1)\right)+2 r_{2} M k \mathrm{e}^{\chi(\phi)}(d+2)\right)},
$$


in which

$$
\begin{aligned}
\chi(\phi) & =N(\phi+C)+\ln \left(\frac{N}{1-M \exp (N(\phi+C))}\right), \\
\phi & =k x+\frac{\lambda^{2}(2 d+1) k}{\alpha \mu\left(d^{3}+5 d^{2}+8 d+4\right)}\left(t+\frac{1}{\Gamma(\alpha)}\right)^{\alpha} .
\end{aligned}
$$

Set V.

$$
\begin{aligned}
L & =0 \\
M & =M \\
N & =N \\
k & =k \\
l & =-\frac{N^{2} k^{3}}{d^{2}}, \\
a_{0} & =\frac{\left(2 M d^{2} r_{2}-N d^{2} q_{2}+6 M d r_{2}-3 N d q_{2}+4 M r_{2}-2 N q_{2}\right) N k^{2} r_{1}}{q_{1} d^{2} \lambda} \\
a_{1} & =-\frac{\left(2 M d^{2} r_{2}-N d^{2} q_{2}+6 M d r_{2}-3 N d q_{2}+4 M r_{2}-2 N q_{2}\right) N k^{2}}{q_{1} d^{2} \lambda} \\
p_{1} & =\frac{M q_{1}}{N}, \\
q_{1} & =q_{1}, \\
q_{2} & =q_{2} \\
r_{1} & =r_{1}, \\
r_{2} & =r_{2} \\
p_{2} & =\frac{4 M k^{2} \mu r_{2}(d+1)(d+2)^{2}\left(M r_{2}-N q_{2}\right)+q_{2}^{2}\left(N^{2} d^{3} k^{2} \mu+5 N^{2} d^{2} k^{2} \mu+8 N^{2} d k^{2} \mu+4 N^{2} k^{2} \mu+2 d^{3} \lambda^{2}+d^{2} \lambda^{2}\right)}{4 d^{2} \lambda^{2} r_{2}(2 d+1)}
\end{aligned}
$$

We, therefore, gained the following generalized solitary solution:

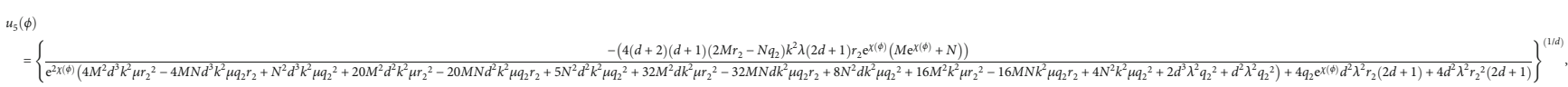


in which

$$
\begin{aligned}
\chi(\phi) & =N(\phi+C)+\ln \left(\frac{N}{1-M \exp (N(\phi+C))}\right), \\
\phi & =k x-\frac{N^{2} k^{3}}{\alpha d^{2}}\left(t+\frac{1}{\Gamma(\alpha)}\right)^{\alpha} .
\end{aligned}
$$

Set VI.

$$
\begin{aligned}
L=0, & =-\frac{(2 d+1) d \lambda p_{1}}{q_{1} k \sqrt{-\mu(d+1)(2 d+1)}(d+2)}, \\
N & =\frac{\sqrt{-\mu(d+1)(2 d+1)} \lambda d}{\mu(d+1)(d+2) k}, \\
l & =\frac{k \lambda^{2}(2 d+1)}{(d+1) \mu(d+2)^{2}}, \\
a_{0} & =\frac{\lambda q_{2}(2 d+1) r_{1}}{\mu q_{1}(d+2)}, \\
a_{1} & =-\frac{\lambda q_{2}(2 d+1)}{\mu q_{1}(d+2)}, \\
p_{1} & =p_{1}, \\
p_{2} & =p_{2}, \\
q_{1} & =q_{1}, \\
q_{2} & =q_{2} \\
r_{1} & =r_{1}, \\
r_{2} & =0 .
\end{aligned}
$$

We, therefore, gained the following generalized solitary solution:

$$
u_{6}(\phi)=\left\{-\frac{\lambda q_{2}(2 d+1)\left(\mathrm{e}^{\chi(\phi)} p_{1}+q_{1}\right)}{\mu q_{1}(d+2)\left(\mathrm{e}^{\chi(\phi)} p_{2}+q_{2}\right)}\right\}^{(1 / d)},
$$

in which

$$
\begin{aligned}
\chi(\phi) & =N(\phi+C)+\ln \left(\frac{N}{1-M \exp (N(\phi+C))}\right) \\
\phi & =k x+\frac{k \lambda^{2}(2 d+1)}{\alpha(d+1) \mu(d+2)^{2}}\left(t+\frac{1}{\Gamma(\alpha)}\right)^{\alpha}
\end{aligned}
$$

Set VII.

$$
\begin{aligned}
& L=0 \text {, } \\
& M=M \text {, } \\
& N=N \text {, } \\
& k=\frac{\sqrt{-\mu(d+1)(2 d+1)} \lambda d}{\mu(d+1)(d+2) N}, \\
& l=\frac{\lambda^{3}(2 d+1) \sqrt{-\mu(d+1)(2 d+1)} d}{\mu^{2}(d+1)\left(d^{3}+5 \quad d^{2}+8 d+4\right)(d+2) N}, \\
& a_{0}=-\frac{\left(M q_{1} r_{2}+N p_{1} r_{2}-N q_{1} q_{2}\right) \lambda(2 d+1) r_{1}}{N(d+2) \mu q_{1}^{2}}, \\
& a_{1}=\frac{\left(M q_{1} r_{2}+N p_{1} r_{2}-N q_{1} q_{2}\right) \lambda(2 d+1)}{N(d+2) \mu q_{1}^{2}}, \\
& p_{1}=p_{1} \text {, } \\
& p_{2}=-\frac{p_{1}\left(p_{1} r_{2}-q_{1} q_{2}\right)}{q_{1}^{2}}, \\
& q_{1}=q_{1}, \\
& q_{2}=q_{2} \text {, } \\
& r_{1}=r_{1} \text {, } \\
& r_{2}=r_{2} \text {. }
\end{aligned}
$$

We, therefore, gained the following generalized solitary solution:

$$
u_{7}(\phi)=\left\{-\frac{\left(M q_{1} r_{2}+N p_{1} r_{2}-N q_{1} q_{2}\right) \lambda(2 d+1) \mathrm{e}^{\chi(\phi)}}{\left(\mathrm{e}^{\chi(\phi)} p_{1} r_{2}-q_{2} \mathrm{e}^{\chi(\phi)} q_{1}-q_{1} r_{2}\right) N(d+2) \mu}\right\}^{(1 / d)}
$$

in which

$$
\begin{aligned}
\chi(\phi)= & N(\phi+C)+\ln \left(\frac{N}{1-M \exp (N(\phi+C))}\right) \\
\phi= & \frac{\sqrt{-\mu(d+1)(2 d+1)} \lambda d}{\mu(d+1)(d+2) N} x \\
& +\frac{\lambda^{3}(2 d+1) \sqrt{-\mu(d+1)(2 d+1)} d}{\alpha \mu^{2}(d+1)\left(d^{3}+5 d^{2}+8 d+4\right)(d+2) N} \\
& \cdot\left(t+\frac{1}{\Gamma(\alpha)}\right)^{\alpha} \cdot
\end{aligned}
$$

Set VIII. 


$$
\begin{aligned}
L & =0, \\
M & =0, \\
N & =\frac{\sqrt{-\mu(d+1)(2 d+1)} \lambda d}{\mu(d+1)(d+2) k}, \\
k & =k, \\
l & =\frac{k \lambda^{2}(2 d+1)}{(d+1) \mu(d+2)^{2}}, \\
a_{0} & =-\frac{d \mu a_{1} r_{1}+2 d \lambda r_{2}+2 \mu a_{1} r_{1}+\lambda r_{2}}{(d+2) \mu}, \\
a_{1} & =a_{1}, \\
p_{1} & =0, \\
p_{2} & =0, \\
q_{1} & =0, \\
q_{2} & =q_{2}, \\
r_{1} & =r_{1}, \\
r_{2} & =r_{2} .
\end{aligned}
$$

We, therefore, gained the following generalized solitary solution:

$$
u_{8}(\phi)=\left\{-\frac{r_{2} \lambda(2 d+1)}{(d+2) \mu\left(q_{2} \mathrm{e}^{\chi(\phi)}+r_{2}\right)}\right\}^{(1 / d)},
$$

in which

$$
\begin{aligned}
\chi(\phi) & =N(\phi+C), \\
\phi & =k x+\frac{k \lambda^{2}(2 d+1)}{\alpha(d+1) \mu(d+2)^{2}}\left(t+\frac{1}{\Gamma(\alpha)}\right)^{\alpha} .
\end{aligned}
$$

Set IX.

$$
\begin{aligned}
L & =0, \\
M & =0, \\
N & =\frac{\sqrt{-\mu(d+1)(2 d+1)} \lambda d}{\mu(d+1)(d+2) k}, \\
k & =k, \\
l & =\frac{k \lambda^{2}(2 d+1)}{(d+1) \mu(d+2)^{2}}, \\
a_{0} & =-\frac{r_{2} \lambda(2 d+1)}{(d+2) \mu}, \\
a_{1} & =0, \\
p_{1} & =p_{1}, \\
p_{2} & =0, \\
q_{1} & =q_{1},
\end{aligned}
$$

$$
\begin{aligned}
& q_{2}=q_{2}, \\
& r_{1}=r_{1}, \\
& r_{2}=r_{2} .
\end{aligned}
$$

We, therefore, gained the following generalized solitary solution:

$$
u_{9}(\phi)=\left\{\frac{a_{1} q_{1} \mathrm{e}^{\chi(\phi)} \mu d+2 a_{1} q_{1} \mathrm{e}^{\chi(\phi)} \mu-2 d \lambda r_{2}-\lambda r_{2}}{(d+2) \mu r_{2}}\right\}^{(1 / d)},
$$

in which

$$
\begin{aligned}
\chi(\phi) & =N(\phi+C), \\
\phi & =k x+\frac{k \lambda^{2}(2 d+1)}{\alpha(d+1) \mu(d+2)^{2}}\left(t+\frac{1}{\Gamma(\alpha)}\right)^{\alpha} .
\end{aligned}
$$

Set $X$.

$$
\begin{aligned}
L & =0, \\
M & =\frac{\sqrt{-\mu\left(2 d^{2}+3 d+1\right)} a_{1} q_{1} d}{\left(2 d^{2}+3 d+1\right) r_{2} k}, \\
N & =\frac{d \lambda\left(2 d^{2}+3 d+1\right)}{(d+2) \sqrt{-\mu\left(2 d^{2}+3 d+1\right)} k(d+1)}, \\
k & =k, \\
l & =\frac{k \lambda^{2}(2 d+1)}{(d+1) \mu(d+2)^{2}}, \\
a_{0} & =-\frac{d \mu a_{1} r_{1}+2 d \lambda r_{2}+2 \mu a_{1} r_{1}+\lambda r_{2}}{(d+2) \mu}, \\
a_{1} & =a_{1}, \\
p_{1} & =0, \\
p_{2} & =0, \\
q_{1} & =q_{1}, \\
q_{2} & =q_{2}, \\
r_{1} & =r_{1}, \\
r_{2} & =r_{2} .
\end{aligned}
$$

We, therefore, gained the following generalized solitary solution:

$$
u_{10}(\phi)=\left\{\frac{a_{1} q_{1} \mathrm{e}^{\chi(\phi)} \mu d+2 a_{1} q_{1} \mathrm{e}^{\chi(\phi)} \mu-2 d \lambda r_{2}-\lambda r_{2}}{(d+2) \mu\left(q_{2} \mathrm{e}^{\chi(\phi)}+r_{2}\right)}\right\}^{(1 / d)},
$$


in which

$$
\begin{gathered}
\chi(\phi)=N(\phi+C)+\ln \left(\frac{N}{1-M \exp (N(\phi+C))}\right), \\
\phi=k x+\frac{k \lambda^{2}(2 d+1)}{\alpha(d+1) \mu(d+2)^{2}}\left(t+\frac{1}{\Gamma(\alpha)}\right)^{\alpha} .
\end{gathered}
$$

Set XI.

$$
\begin{aligned}
L & =0, \\
M & =0, \\
N & =\frac{\sqrt{-\mu(d+1)(2 d+1)} \lambda d}{\mu(d+1)(d+2) k}, \\
k & =k, \\
l & =\frac{k \lambda^{2}(2 d+1)}{(d+1) \mu(d+2)^{2}}, \\
a_{0} & =-\frac{d \mu a_{1} r_{1}+2 d \lambda r_{2}+2 \mu a_{1} r_{1}+\lambda r_{2}}{(d+2) \mu}, \\
a_{1} & =a_{1}, \\
p_{1} & =0, \\
p_{2} & =p_{2}, \\
q_{1} & =q_{1}, \\
q_{2} & =-\frac{d^{2} \mu^{2} a_{1}{ }^{2} q_{1}{ }^{2}+4 d \mu^{2} a_{1}{ }^{2} q_{1}{ }^{2}+4 d^{2} \lambda^{2} p_{2} r_{2}+4 \mu^{2} a_{1}^{2} q_{1}{ }^{2}+4 d \lambda^{2} p_{2} r_{2}+\lambda^{2} p_{2} r_{2},}{\mu\left(2 d^{2}+5 d+2\right) a_{1} q_{1} \lambda} \\
r_{1} & =r_{1}, \\
r_{2} & =r_{2} .
\end{aligned}
$$

We, therefore, gained the following generalized solitary solution:

$$
u_{11}(\phi)=\left\{\frac{\lambda q_{1} a_{1}(2 d+1)}{2 \mathrm{e}^{\chi(\phi)} d \lambda p_{2}-d \mu a_{1} q_{1}+\mathrm{e}^{\chi(\phi)} \lambda p_{2}-2 \mu a_{1} q_{1}}\right\}^{(1 / d)},
$$

in which

$$
\begin{aligned}
\chi(\phi) & =N(\phi+C), \\
\phi & =k x+\frac{k \lambda^{2}(2 d+1)}{\alpha(d+1) \mu(d+2)^{2}}\left(t+\frac{1}{\Gamma(\alpha)}\right)^{\alpha} .
\end{aligned}
$$

Set XII.

$$
\begin{aligned}
L & =0, \\
M & =-\frac{1}{4} \frac{q_{2} d \lambda(2 d+1)}{r_{2} k \sqrt{-\mu(d+1)(2 d+1)}(d+2)}, \\
N & =\frac{1}{2} \frac{\sqrt{-\mu(d+1)(2 d+1)}}{\mu(d+1)}, \\
k & =k, \\
l & =\frac{k \lambda^{2}(2 d+1)}{(d+1) \mu(d+2)^{2}},
\end{aligned}
$$

$$
\begin{aligned}
& a_{0}=-\frac{d \mu a_{1} r_{1}+2 d \lambda r_{2}+2 \mu a_{1} r_{1}+\lambda r_{2}}{(d+2) \mu}, \\
& a_{1}=a_{1}, \\
& p_{1}=-\frac{1}{4} \frac{(2 d+1) q_{2}{ }^{2} \lambda}{(d+2) a_{1} r_{2} \mu}, p_{2}=p_{2}, \\
& q_{1}=-\frac{q_{2} \lambda(2 d+1)}{(d+2) a_{1} \mu}, \\
& q_{2}=q_{2}, \\
& r_{1}=r_{1}, \\
& r_{2}=r_{2} .
\end{aligned}
$$

We, therefore, gained the following generalized solitary solution:

$$
\begin{aligned}
& u_{12}(\phi) \\
& =\left\{-\frac{1 \lambda}{4} \frac{\lambda\left(2 q_{2}{ }^{2} \mathrm{e}^{2 \chi(\phi)} d+q_{2}{ }^{2} \mathrm{e}^{2 \chi(\phi)}+8 q_{2} \mathrm{e}^{\chi(\phi)} r_{2} d+4 q_{2} \mathrm{e}^{\chi(\phi)} r_{2}+8 d r_{2}{ }^{2}+4 r_{2}{ }^{2}\right)}{\left(p_{2} \mathrm{e}^{2 \chi(\phi)}+q_{2} \mathrm{e}^{\chi(\phi)}+r_{2}\right)(d+2) r_{2} \mu}\right\}^{(1 / d)},
\end{aligned}
$$

in which

$$
\begin{gathered}
\chi(\phi)=N(\phi+C)+\ln \left(\frac{N}{1-M \exp (N(\phi+C))}\right), \\
\phi=k x+\frac{k \lambda^{2}(2 d+1)}{\alpha(d+1) \mu(d+2)^{2}}\left(t+\frac{1}{\Gamma(\alpha)}\right)^{\alpha} .
\end{gathered}
$$

Case II. Then the exact solution will be as

$$
v(\eta)=\frac{\sinh (\chi(\phi)) a_{1} p_{1}+\cosh (\chi(\phi)) a_{1} q_{1}+a_{1} r_{1}+a_{0}}{p_{2} \sinh (\chi(\phi))+q_{2} \cosh (\chi(\phi))+r_{2}} .
$$

Inserting (56) into equation (16), we obtain

$$
\begin{aligned}
& \left(d^{2}(d+1)(2 d+1)\left(p_{2} \sinh (\chi(\phi))+q_{2} \cosh (\chi(\phi))+r_{2}\right)^{4}\right)^{-1} \\
& \cdot \sum_{i+j=6} C_{i j} \sinh ^{i}(\chi(\phi)) \cosh ^{j}(\chi(\phi))=0
\end{aligned}
$$

where $C_{i j}(i+j=6,0 \leq i, j \leq 6)$ are polynomial statements in terms of $a_{0}, a_{1}, p_{1}, p_{2}, q_{1}, q_{2}, r_{1}$, and $r_{2}$. Hence, solving the resulting system $C_{i j}=0(i+j=6,0 \leq i, j \leq 6)$ simultaneously, we acquire the following sets of parameters of solutions. 
Set I.

$$
\begin{aligned}
L & =0, \\
M & =0, \\
N & =\frac{1}{2} \frac{\sqrt{-\mu(d+1)(2 d+1)} \lambda d}{\mu(d+1)(d+2) k}, \\
k & =k, \\
l & =\frac{\lambda^{2}(2 d+1) k}{\mu\left(d^{3}+5 d^{2}+8 d+4\right)}, \\
a_{0} & =-\frac{r_{2} \lambda(2 d+1)}{(d+2) \mu}, \\
a_{1} & =0, \\
p_{1} & =p_{1}, \\
p_{2} & =q_{2}, \\
q_{1} & =q_{1}, \\
q_{2} & =q_{2}, \\
r_{1} & =r_{1}, \\
r_{2} & =r_{2} .
\end{aligned}
$$

We, therefore, gained the following generalized solitary solution:

$$
\begin{aligned}
u_{1}(\phi) & =\left(-\frac{\lambda(2 d+1) r_{2}}{\mu(d+2)\left(q_{2} \sinh (\chi(\phi))+q_{2} \cosh (\chi(\phi))+r_{2}\right)}\right)^{(1 / d)}, \\
\chi(\phi) & =\frac{1}{2} \frac{\sqrt{-\mu(d+1)(2 d+1)} \lambda d}{\mu(d+1)(d+2) k}(\phi+C),
\end{aligned}
$$

in which

$$
\phi=k x+\frac{\lambda^{2}(2 d+1) k}{\mu \alpha\left(d^{3}+5 d^{2}+8 d+4\right)}\left(t+\frac{1}{\Gamma(\alpha)}\right)^{\alpha} .
$$

Set II.

$$
\begin{aligned}
L & =0 \\
M & =0 \\
N & =\frac{\sqrt{-\mu(d+1)(2 d+1)} \lambda d}{\mu(d+1)(d+2) k} \\
k & =k, \\
l & =\frac{\lambda^{2}(2 d+1) k}{\mu\left(d^{3}+5 d^{2}+8 d+4\right)}, \\
a_{0} & =-\frac{r_{2} \lambda(2 d+1)}{(d+2) \mu} \\
a_{1} & =0 \\
p_{1} & =p_{1} \\
p_{2} & =-q_{2}
\end{aligned}
$$

$$
\begin{aligned}
& q_{1}=q_{1}, \\
& q_{2}=q_{2}, \\
& r_{1}=r_{1}, \\
& r_{2}=r_{2} .
\end{aligned}
$$

We, therefore, gained the following generalized solitary solution:

$$
\begin{aligned}
u_{2}(\phi) & =\left(\frac{\lambda(2 d+1) r_{2}}{\mu(d+2)\left(q_{2} \sinh (\chi(\phi))-q_{2} \cosh (\chi(\phi))-r_{2}\right)}\right)^{(1 / d)}, \\
\chi(\phi) & =\frac{\sqrt{-\mu(d+1)(2 d+1)} \lambda d}{\mu(d+1)(d+2) k}(\phi+C),
\end{aligned}
$$

in which

$$
\phi=k x+\frac{\lambda^{2}(2 d+1) k}{\mu \alpha\left(d^{3}+5 d^{2}+8 d+4\right)}\left(t+\frac{1}{\Gamma(\alpha)}\right)^{\alpha} .
$$

Set III.

$$
\begin{aligned}
L & =0, \\
M & =0, \\
N & =\frac{1}{2} \frac{\sqrt{-\mu(d+1)(2 d+1)} \lambda d}{\mu(d+1)(d+2) k}, \\
k & =k, \\
l & =\frac{\lambda^{2}(2 d+1) k}{\mu\left(d^{3}+5 d^{2}+8 d+4\right)}, \\
a_{0} & =\frac{1}{2} \frac{\lambda\left(2 d p_{2}+2 d q_{2}+p_{2}+q_{2}\right) r_{1}}{q_{1}(d+2) \mu}, \\
a_{1} & =-\frac{1}{2} \frac{\lambda\left(2 d p_{2}+2 d q_{2}+p_{2}+q_{2}\right)}{q_{1}(d+2) \mu}, \\
p_{1} & =q_{1}, \\
p_{2} & =p_{2}, \\
q_{1} & =q_{1}, \\
q_{2} & =q_{2}, \\
r_{1} & =r_{1}, \\
r_{2} & =0 .
\end{aligned}
$$

We, therefore, gained the following generalized solitary solution:

$$
\begin{aligned}
& u_{3}(\phi)=\left(-\frac{1}{2} \frac{\lambda\left(2 d p_{2}+2 d q_{2}+p_{2}+q_{2}\right)(\sinh (\chi(\phi))+\cosh (\chi(\phi)))}{\mu(d+2)\left(p_{2} \sinh (\chi(\phi))+q_{2} \cosh (\chi(\phi))\right)}\right)^{(1 / d)}, \\
& \chi(\phi)=\frac{1}{2} \frac{\sqrt{-\mu(d+1)(2 d+1)} \lambda d}{\mu(d+1)(d+2) k}(\phi+C),
\end{aligned}
$$

in which 


$$
\phi=k x+\frac{\lambda^{2}(2 d+1) k}{\mu \alpha\left(d^{3}+5 d^{2}+8 d+4\right)}\left(t+\frac{1}{\Gamma(\alpha)}\right)^{\alpha} .
$$

Set IV.

$$
\begin{aligned}
L & =0, \\
M & =0, \\
N & =\frac{1}{2} \frac{\sqrt{-\mu(d+1)(2 d+1)} \lambda d}{\mu(d+1)(d+2) k}, \\
k & =k, \\
l & =\frac{\lambda^{2}(2 d+1) k}{\mu\left(d^{3}+5 d^{2}+8 d+4\right)}, \\
a_{0} & =\frac{1}{2} \frac{\lambda\left(2 d p_{2}-2 d q_{2}+p_{2}-q_{2}\right) r_{1}}{q_{1}(d+2) \mu}, \\
a_{1} & =-\frac{1}{2} \frac{\lambda\left(2 d p_{2}-2 d q_{2}+p_{2}-q_{2}\right)}{q_{1}(d+2) \mu}, \\
p_{1} & =-q_{1}, \\
p_{2} & =p_{2}, \\
q_{1} & =q_{1}, \\
q_{2} & =q_{2}, \\
r_{1} & =r_{1}, \\
r_{2} & =0 .
\end{aligned}
$$

We, therefore, gained the following generalized solitary solution:

$$
\begin{aligned}
u_{4}(\phi) & =\left(-\frac{1}{2} \frac{\lambda\left(2 d p_{2}-2 d q_{2}+p_{2}-q_{2}\right)(\sinh (\chi(\phi))-\cosh (\chi(\phi)))}{\mu(d+2)\left(p_{2} \sinh (\chi(\phi))+q_{2} \cosh (\chi(\phi))\right)}\right)^{(1 / d)}, \\
\chi(\phi) & =\frac{1}{2} \frac{\sqrt{-\mu(d+1)(2 d+1)} \lambda d}{\mu(d+1)(d+2) k}(\phi+C),
\end{aligned}
$$

in which

$$
\phi=k x+\frac{\lambda^{2}(2 d+1) k}{\mu \alpha\left(d^{3}+5 d^{2}+8 d+4\right)}\left(t+\frac{1}{\Gamma(\alpha)}\right)^{\alpha} .
$$

Set $V$.

$$
\begin{aligned}
L & =0, \\
M & =0 \\
N & =\frac{1}{2} \frac{\sqrt{-\mu(d+1)(2 d+1)} \lambda d}{\mu(d+1)(d+2) k}, \\
k & =k, \\
l & =\frac{\lambda^{2}(2 d+1) k}{\mu\left(d^{3}+5 d^{2}+8 d+4\right)}, \\
a_{0} & =-\frac{1}{2} \frac{q_{2} \lambda(2 d+1) r_{1}}{\mu p_{1}(d+2)}
\end{aligned}
$$

$$
\begin{aligned}
& a_{1}=\frac{1}{2} \frac{q_{2} \lambda(2 d+1)}{\mu p_{1}(d+2)}, \\
& p_{1}=p_{1}, \\
& p_{2}=0 \\
& q_{1}=-p_{1}, \\
& q_{2}=q_{2}, \\
& r_{1}=r_{1}, \\
& r_{2}=0 .
\end{aligned}
$$

We, therefore, gained the following generalized solitary solution:

$$
\begin{aligned}
u_{5}(\phi) & =\left(\frac{1}{2} \frac{(2 d+1) \lambda(\sinh (\chi(\phi))-\cosh (\chi(\phi)))}{\mu(d+2) \cosh (\chi(\phi))}\right)^{(1 / d)}, \\
\chi(\phi) & =\frac{1}{2} \frac{\sqrt{-\mu(d+1)(2 d+1)} \lambda d}{\mu(d+1)(d+2) k}(\phi+C)
\end{aligned}
$$

in which

$$
\phi=k x+\frac{\lambda^{2}(2 d+1) k}{\mu \alpha\left(d^{3}+5 d^{2}+8 d+4\right)}\left(t+\frac{1}{\Gamma(\alpha)}\right)^{\alpha} .
$$

Set VI.

$$
\begin{aligned}
L & =0, \\
M & =0, \\
N & =\frac{1}{2} \frac{\sqrt{-\mu(d+1)(2 d+1)} \lambda d}{\mu(d+1)(d+2) k}, \\
k & =k, \\
l & =\frac{\lambda^{2}(2 d+1) k}{\mu\left(d^{3}+5 d^{2}+8 \quad d+4\right)}, \\
a_{0} & =\frac{1}{2} \frac{q_{2} \lambda(2 d+1) r_{1}}{\mu p_{1}(d+2)}, \\
a_{1} & =-\frac{1}{2} \frac{q_{2} \lambda(2 d+1)}{\mu p_{1}(d+2)}, \\
p_{1} & =p_{1}, \\
p_{2} & =0, \\
q_{1} & =p_{1}, \\
q_{2} & =q_{2}, \\
r_{1} & =r_{1}, \\
r_{2} & =0 .
\end{aligned}
$$




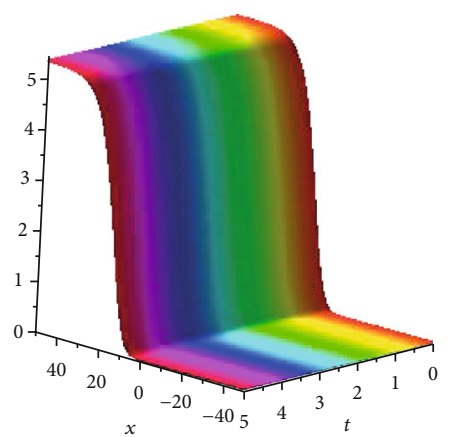

(a)

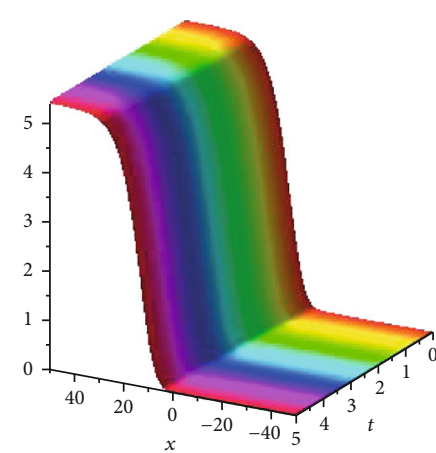

(c)

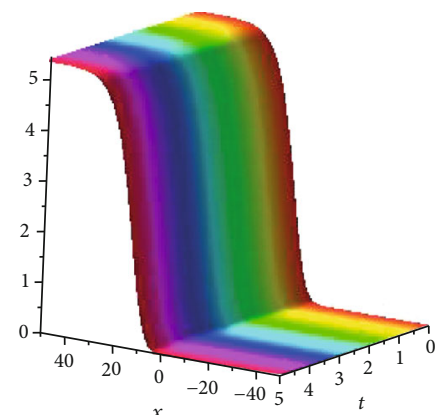

(b)

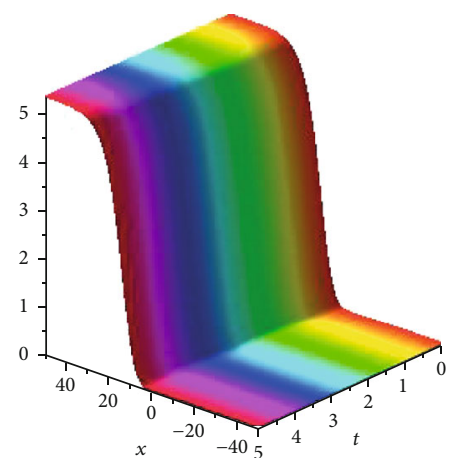

(d)

Figure 1: The 3D plot of (21) at $d=0.2, \mu=-1, p_{2}=1.5, r_{2}=2, \lambda=2.2$, and $k=3$ when (a) $\alpha=0.25$, (b) $\alpha=0.5$, (c) $\alpha=0.85$, and (d) $\alpha=0.99$.

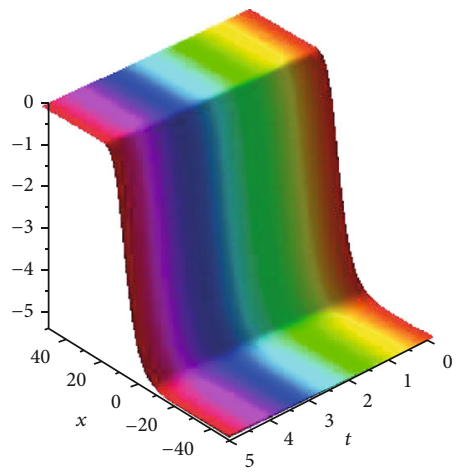

(a)

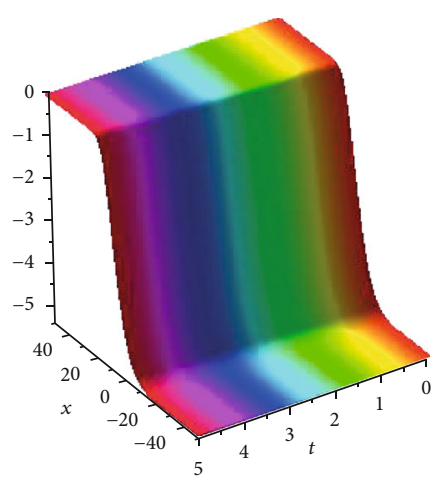

(c)

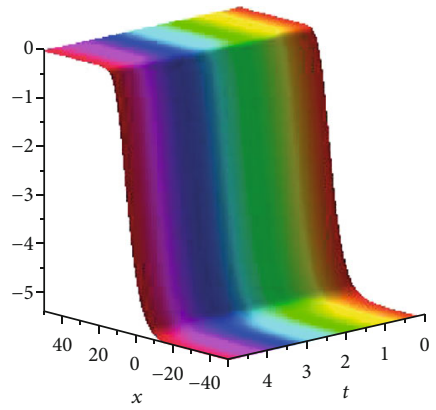

(b)

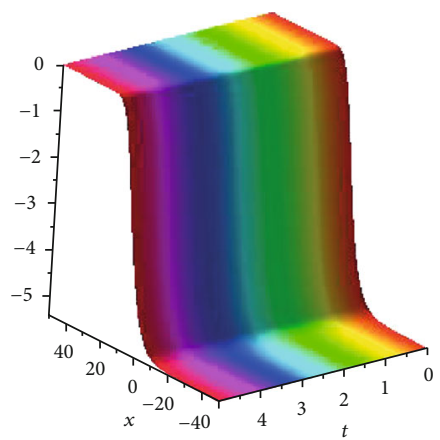

(d)

Figure 2: The 3D plot of (27) at $d=0.2, \mu=-1, p_{2}=1.5, q_{2}=2, \lambda=2.2, M=-3, N=2$, and $k=3$ when (a) $\alpha=0.25$, (b) $\alpha=0.5,(\mathrm{c}) \alpha=0.85$, and (d) $\alpha=0.99$. 


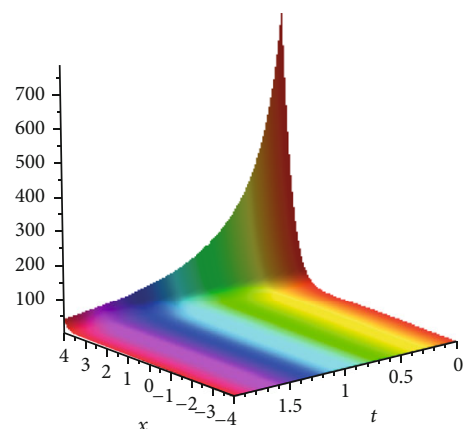

(a)

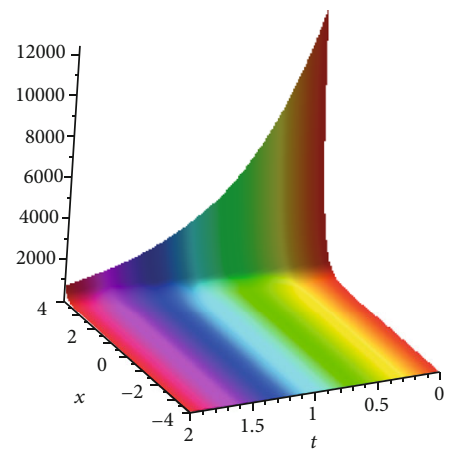

(c)

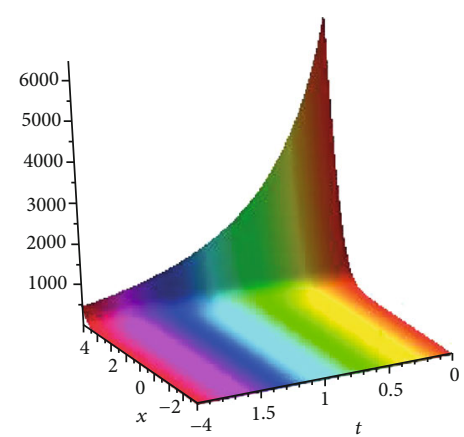

(b)

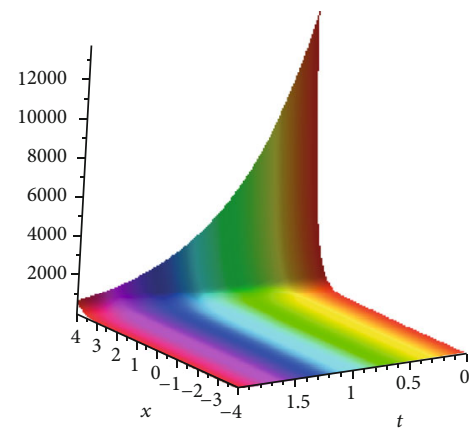

(d)

Figure 3: The 3D plot of (45) at $d=2, \mu=-1, a_{1}=1.5, q_{1}=0.2, r_{2}=2, \lambda=2.2, M=-3, N=2$, and $k=3$ when (a) $\alpha=0.25$, (b) $\alpha=0.5$, (c) $\alpha=0.85$, and (d) $\alpha=0.99$.

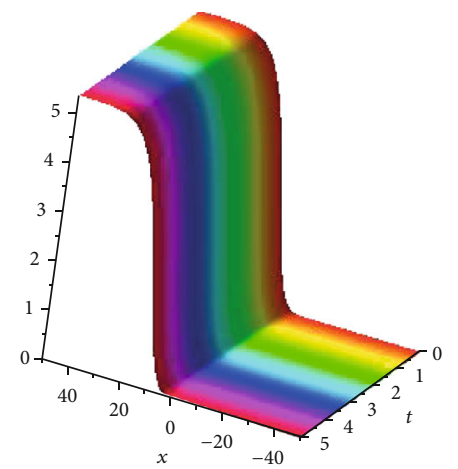

(a)

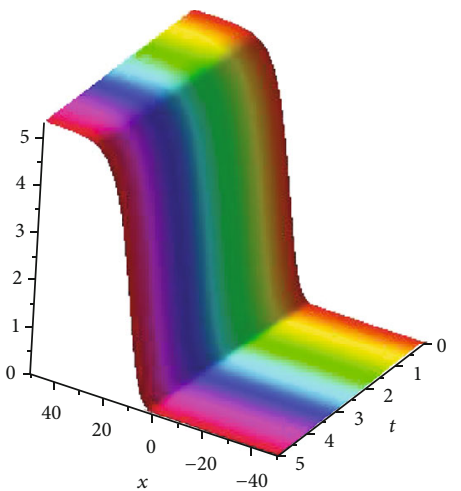

(c)

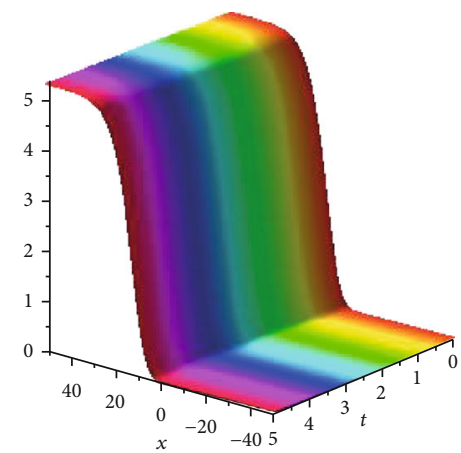

(b)

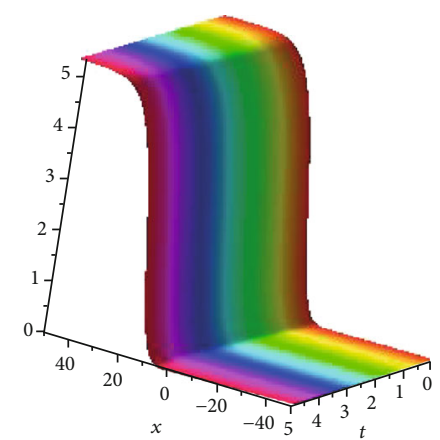

(d)

Figure 4: The 3D plot of (59) at $d=0.2, \mu=-1, q_{2}=1.5, r_{2}=2, \lambda=2.2, M=3, N=2$, and $k=3$ when (a) $\alpha=0.25$, (b) $\alpha=0.5$, (c) $\alpha=0.85$, and (d) $\alpha=0.99$. 


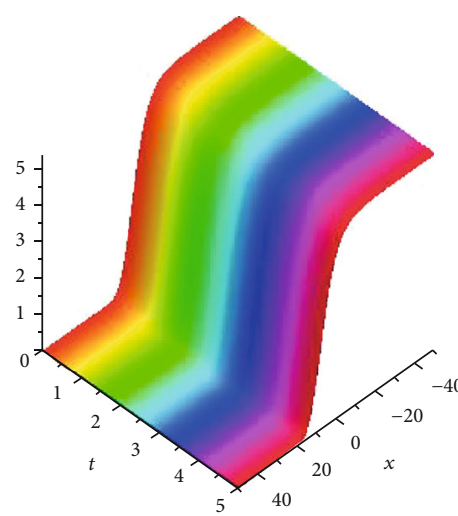

(a)

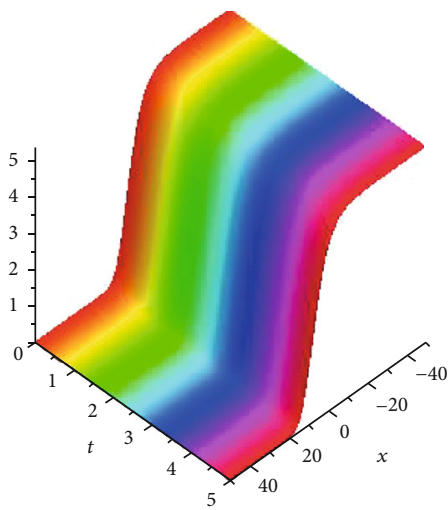

(c)

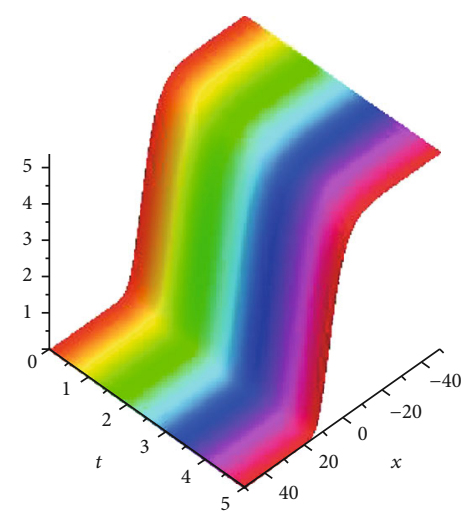

(b)

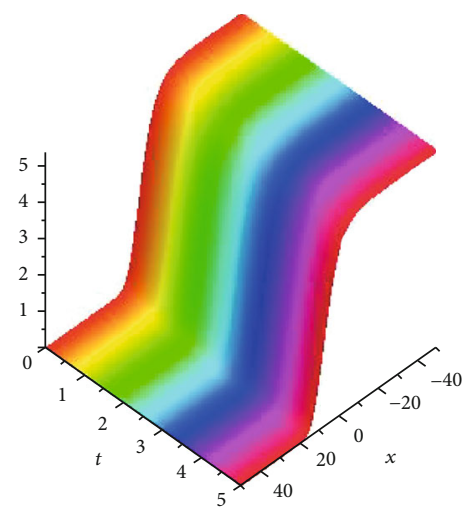

(d)

Figure 5: The 3D plot of (65) at $d=0.2, \mu=-1, p_{2}=1.5, q_{2}=2, \lambda=2.2, M=3, N=2$, and $k=3$ when (a) $\alpha=0.25$, (b) $\alpha=0.5$, (c) $\alpha=0.85$, and (d) $\alpha=0.99$.

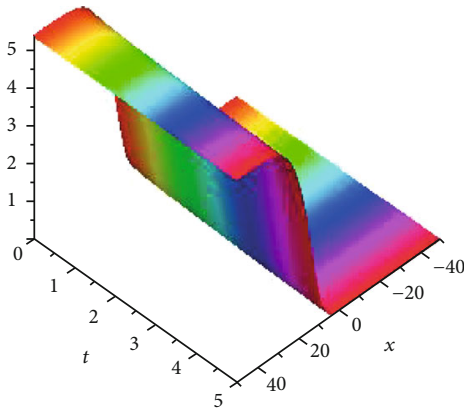

(a)

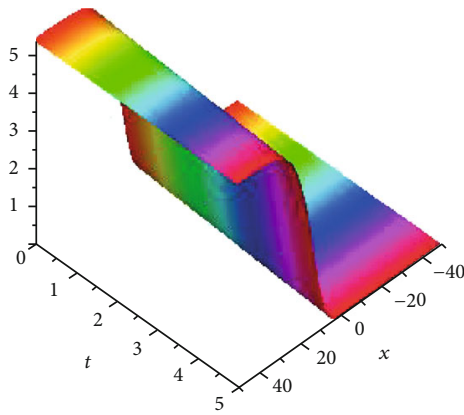

(c)

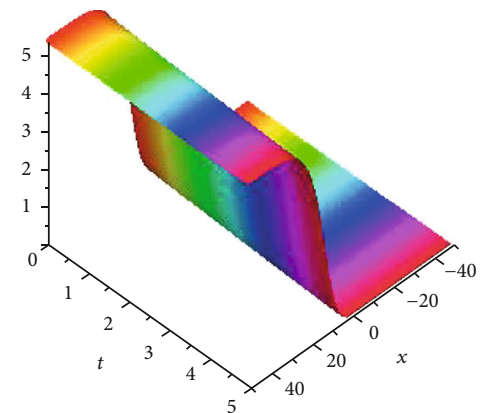

(b)

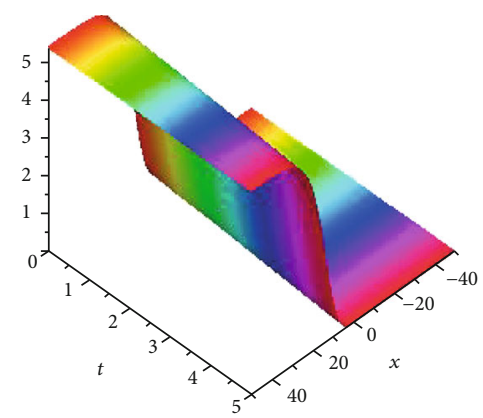

(d)

Figure 6: The 3D plot of (80) at $d=0.2, \mu=-1, r_{2}=1.5, q_{2}=2, \lambda=2.2, M=3, N=2$, and $k=3$, when (a) $\alpha=0.25$, (b) $\alpha=0.5$, (c) $\alpha=0.85$, and (d) $\alpha=0.99$. 
We, therefore, gained the following generalized solitary solution:

$$
\begin{aligned}
u_{6}(\phi) & =\left(-\frac{1}{2} \frac{(2 d+1) \lambda(\sinh (\chi(\phi))+\cosh (\chi(\phi)))}{\mu(d+2) \cosh (\chi(\phi))}\right)^{(1 / d)}, \\
\chi(\phi) & =\frac{1}{2} \frac{\sqrt{-\mu(d+1)(2 d+1)} \lambda d}{\mu(d+1)(d+2) k}(\phi+C),
\end{aligned}
$$

in which

$$
\phi=k x+\frac{\lambda^{2}(2 d+1) k}{\mu \alpha\left(d^{3}+5 d^{2}+8 d+4\right)}\left(t+\frac{1}{\Gamma(\alpha)}\right)^{\alpha} .
$$

Set VII.

$$
\begin{aligned}
L & =0 \\
M & =0 \\
N & =\frac{1}{2} \frac{\sqrt{-\mu(d+1)(2 d+1)} \lambda d}{\mu(d+1)(d+2) k}, \\
k & =k, \\
l & =\frac{\lambda^{2}(2 d+1) k}{\mu\left(d^{3}+5 d^{2}+8 \quad d+4\right)}, \\
a_{0} & =\frac{1}{2} \frac{q_{2} \lambda(2 d+1) r_{1}}{\mu p_{1}}, \\
a_{1} & =-\frac{1}{2} \frac{q_{2} \lambda(2 d+1)}{\mu p_{1}}, \\
p_{1} & =p_{1}, \\
p_{2} & =(d+1) q_{2}, \\
q_{1} & =p_{1}, \\
q_{2} & =q_{2}, \\
r_{1} & =r_{1}, \\
r_{2} & =0 .
\end{aligned}
$$

We, therefore, gained the following generalized solitary solution:

$$
\begin{aligned}
u_{7}(\phi) & =\left(-\frac{1}{2} \frac{(2 d+1) \lambda(\sinh (\chi(\phi))+\cosh (\chi(\phi)))}{\mu(\sinh (\chi(\phi)) d+\sinh (\chi(\phi))+\cosh (\chi(\phi)))}\right)^{(1 / d)}, \\
\chi(\phi) & =\frac{1}{2} \frac{\sqrt{-\mu(d+1)(2 d+1)} \lambda d}{\mu(d+1)(d+2) k}(\phi+C),
\end{aligned}
$$

in which

$$
\phi=k x+\frac{\lambda^{2}(2 d+1) k}{\mu \alpha\left(d^{3}+5 d^{2}+8 d+4\right)}\left(t+\frac{1}{\Gamma(\alpha)}\right)^{\alpha} .
$$

Set VIII.

$$
\begin{aligned}
L & =0, \\
M & =0, \\
N & =\frac{1}{2} \frac{\sqrt{-\mu(d+1)(2 d+1)} \lambda d}{\mu(d+1)(d+2) k}, \\
k & =k, \\
l & =\frac{\lambda^{2}(2 d+1) k}{\mu\left(d^{3}+5 d^{2}+8 d+4\right)}, \\
a_{0} & =-\frac{1}{2} \frac{q_{2} \lambda(2 d+1) r_{1}}{\mu p_{1}}, \\
a_{1} & =\frac{1}{2} \frac{q_{2} \lambda(2 d+1)}{\mu p_{1}}, \\
p_{1} & =p_{1}, \\
p_{2} & =-(d+1) q_{2}, \\
q_{1} & =-p_{1}, \\
q_{2} & =q_{2} \\
r_{1} & =r_{1}, \\
r_{2} & =0 .
\end{aligned}
$$

We, therefore, gained the following generalized solitary solution:

$$
\begin{aligned}
& u_{8}(\phi)=\left(-\frac{1}{2} \frac{(2 d+1) \lambda(\sinh (\chi(\phi))-\cosh (\chi(\phi)))}{\mu(\sinh (\chi(\phi)) d+\sinh (\chi(\phi))-\cosh (\chi(\phi)))}\right)^{(1 / d)} \\
& \chi(\phi)=\frac{1}{2} \frac{\sqrt{-\mu(d+1)(2 d+1)} \lambda d}{\mu(d+1)(d+2) k}(\phi+C)
\end{aligned}
$$

in which

$$
\phi=k x+\frac{\lambda^{2}(2 d+1) k}{\mu \alpha\left(d^{3}+5 d^{2}+8 d+4\right)}\left(t+\frac{1}{\Gamma(\alpha)}\right)^{\alpha} .
$$

\section{Graphical Representation}

The graphical description of derived soliton and other solutions have been expressed in the mentioned figures by allotting the different values of the parameters. The $3 \mathrm{D}$ plots for some solutions of the considered equation for four fractional order cases $\alpha=0.25,0.5,0.85$, and 0.99 have been shown. When all obtained exact solutions for the fractional 
generalized Korteweg-de Vries equation are examined, the exact solution (32) is similar to the solution of Sahadevan and Bakkyaraj [64], the solution of Li et al. [65] (48), and the solution of Akbulut and Tascan [66] (74) in the literature. There are other obtained exact solutions that are not included in the literature, and it can be said that they are new exact solutions obtained by the new version trial equation method. The results are new, interesting, and have a great impact in the field of nonlinear sciences where the $(2+1)$-dimensional $\mathrm{KdV}$ equation will be used for the dynamics of nonlinear solitons and other solutions. Also, two- and three-dimensional graphics of the obtained solution functions are illustrated in Figures 1-6 which demonstrate suitable parametric choices.

\section{Conclusion}

We used the direct truncation method to find the explicit solution of the fractional generalized Korteweg-de Vries equation, comparing it with the known results in the literature, and we were able to get some novel solitary wave solutions, including bright, dark, kink, and periodic solitons. Numerical simulation has been performed by utilizing the Maple software. The solution of each PDE is always utilized for understanding the system and various phenomena described by it. The new analytical expansion method is helpful for obtaining the solutions in the form of hyperbolic and ergometric forms which are exact and helpful in understanding its fractional forms. It is not hard to see that the general solution is an algebraically localized wave decayed in all space directions and existing at all times. Finally, a transformation is used to draw soliton solutions of equation (1) using the Maple software. The 3D plot for some solutions of the considered equation for four fractional order cases $\alpha=0.25,0.5$ , 0.85, and 0.99 are shown in Figures 1-6. Finally, we show some graphs to explain these solutions. So, this gives the efficient applications of the new analytical expansion for the fractional PDEs. In future works with the generalized expansion function based on combined functions, we next aim at the fractional generalized $\mathrm{KdV}$ equation or nonlinear wave equations, which possess more terms and higher nonlinearity than the standard equations.

\section{Data Availability}

The datasets supporting the conclusions of article are included within the article and its additional file.

\section{Disclosure}

The submission of the manuscript is in the preprint.

\section{Conflicts of Interest}

The authors declare that they have no conflict of interest.

\section{References}

[1] C. Schmeiser and K. Fellnerand, "Burgers-Poisson: a nonlinear dispersive model equation," SIAM Journal on Applied Mathematics, vol. 64, no. 5, pp. 1509-1525, 2004.

[2] M. Gaur and K. Singh, "On group invariant solutions of fractional order Burgers-Poisson equation," Applied Mathematics and Computation, vol. 244, pp. 870-877, 2014.

[3] M. Kato, "Large time behavior of solutions to the BurgersPoisson equations," Osaka Journal of Mathematics, vol. 4, pp. 923-943, 2007.

[4] S. Momani, "An explicit and numerical solutions of the fractional KdV equation," Mathematics and Computers in Simulation, vol. 70, no. 2, pp. 110-118, 2005.

[5] M. Antonova and A. Biswas, "Adiabatic parameter dynamics of perturbed solitary waves," Communications in Nonlinear Science and Numerical Simulation, vol. 14, no. 3, pp. 734$748,2009$.

[6] J. He, "Nonlinear oscillation with fractional derivative and its applications," in International Conference on Vibrating Engineering, pp. 288-291, Dalian, China, 1998.

[7] J. He, "Some applications of nonlinear fractional differential equations and their approximations," Bulletin of Science, Technology \& Society, vol. 15, pp. 86-90, 1999.

[8] J. He, "Approximate analytical solution for seepage flow with fractional derivatives in porous media," Computer Methods in Applied Mechanics and Engineering, vol. 167, no. 1-2, pp. 57-68, 1998.

[9] I. Grigorenko and E. Grigorenko, "Chaotic dynamics of the fractional lorenz system," Physical Review Letters, vol. 91, no. 3, article 034101, 2003.

[10] S. Momani and N. T. Shawagfeh, "Decomposition method for solving fractional Riccati differential equations," Applied Mathematics and Computation, vol. 182, no. 2, pp. 10831092, 2006.

[11] S. Momani and M. A. Noor, "Numerical methods for fourthorder fractional integro-differential equations," Applied Mathematics and Computation, vol. 182, no. 1, pp. 754-760, 2006.

[12] V. Daftardar-Gejji and H. Jafari, "Solving a multi-order fractional differential equation using Adomian decomposition," Applied Mathematics and Computation, vol. 189, no. 1, pp. 541-548, 2007.

[13] O. Guner, A. Bekir, and H. Bilgil, "A note on exp-function method combined with complex transform method applied to fractional differential equations," Advances in Nonlinear Analysis, vol. 4, no. 3, pp. 201-208, 2015.

[14] S. Momani and Z. Odibat, "Homotopy perturbation method for nonlinear partial differential equations of fractional order," Physics Letters A, vol. 365, no. 5-6, pp. 345-350, 2007.

[15] N. H. Sweilam, M. M. Khader, and R. F. Al-Bar, "Numerical studies for a multi-order fractional differential equation," Physics Letters A, vol. 371, no. 1-2, pp. 26-33, 2007.

[16] B. Zheng and C. Wen, "Exact solutions for fractional partial differential equations by a new fractional sub-equation method," Advances in Difference Equations, vol. 2013, no. 1, 2013.

[17] B. Lu, "The first integral method for some time fractional differential equations," Journal of Mathematical Analysis and Applications, vol. 395, no. 2, pp. 684-693, 2012.

[18] H. Jafari and S. Seifi, "Solving a system of nonlinear fractional partial differential equations using homotopy analysis 
method," Communications in Nonlinear Science and Numerical Simulation, vol. 14, no. 5, pp. 1962-1969, 2009.

[19] A. Bekir, O. Guner, A. H. Bhrawy, and A. Biswas, "Solving nonlinear fractional differential equations using exp-function and $\left(G^{\prime} / G\right)$-expansion methods," Romanian Journal of Physics, vol. 60, no. 3-4, pp. 360-378, 2015.

[20] M. A. E. Herzallah and K. A. Gepreel, "Approximate solution to the time-space fractional cubic nonlinear Schrodinger equation," Applied Mathematical Modelling, vol. 36, no. 11, pp. 5678-5685, 2012.

[21] M. S. Mohamed, "Analytical treatment of Abel integral equations by optimal homotopy analysis transform method," Journal of Information and Computing Science, vol. 10, no. 1, pp. 19-28, 2015.

[22] K. A. Gepreel and M. S. Mohamed, "An optimal homotopy analysis method nonlinear fractional differential equation," Journal of Advanced Research in Dynamical and Control Systems, vol. 6, no. 1, pp. 1-10, 2014.

[23] S. Kemple and H. Beyer, "Global and causal solutions of fractional differential equations, transform methods and special functions: Varna 96," in Proceedings of 2nd international workshop (SCTP), vol. 96, pp. 210-216, Singapore, 1997.

[24] S. Arshed, "Two reliable techniques for the soliton solutions of perturbed Gerdjikov-Ivanov equation," Optik, vol. 164, pp. 93-99, 2018.

[25] A. Biswas, Y. Yildirim, E. Yasar et al., "Optical soliton perturbation with Gerdjikov-Ivanov equation by modified simple equation method," Optik, vol. 157, pp. 1235-1240, 2018.

[26] A. Biswas and R. T. Alqahtani, "Chirp-free bright optical solitons for perturbed Gerdjikov-Ivanov equation by semi-inverse variational principle," Optik, vol. 147, pp. 72-76, 2017.

[27] A. Biswas, Y. Yıldırım, E. Yaşar et al., "Solitons for perturbed Gerdjikov-Ivanov equation in optical fibers and PCF by extended Kudryashov's method," Optical and Quantum Electronics, vol. 50, no. 3, p. 1, 2018.

[28] E. Yasar and Y. Yildirim, "New optical solitons of space-time conformable fractional perturbed Gerdjikov-Ivanov equation by sine-Gordon equation method," Results in Physics, vol. 9, pp. 1666-1672, 2018.

[29] A. Biswas, M. Ekici, A. Sonmezoglu et al., "Optical soliton perturbation for Gerdjikov-Ivanov equation by extended trial equation method," Optik, vol. 158, pp. 747-752, 2018.

[30] I. Podlubny, Fractional Differential Equations: An Introduction to Fractional Derivatives, Fractional Differential Equations, to Methods of Their Solution and Some of Their Applications, Academic Press, New York, 1999.

[31] S. G. Samko, A. A. Kilbas, and O. I. Marichev, Fractional Integrals and Derivatives: Theory and Applications, Gordon and Breach, Yverdon, 1993.

[32] L. Debanth, "Recent applications of fractional calculus to science and engineering," International Journal of Mathematics and Mathematical Sciences, vol. 54, 3442 pages, 2003.

[33] V. E. Zakharov and E. A. Kuznetsov, "On three-dimensional solitons," Soviet Physics, vol. 39, pp. 285-288, 1974.

[34] J. H. He and X. H. Wu, "Exp-function method for nonlinear wave equations," Chaos, Solitons \& Fractals, vol. 30, no. 3, pp. 700-708, 2006.

[35] M. A. Abdou, "Generalized solitonary and periodic solutions for nonlinear partial differential equations by the Expfunction method," Nonlinear Dynamics, vol. 52, no. 1-2, pp. 1-9, 2008.
[36] A. Boz and A. Bekir, "Application of Exp-function method for $(3+1)$-dimensional nonlinear evolution equations," Computers \& Mathematcs with Applications, vol. 56, no. 5, pp. 1451-1456, 2008.

[37] R. Y. Molliq and B. Batiha, "solutions of fractional ZakharovKuznetsov equations by FCT method; this paper has been withdrawn," International Journal of Engineering \& Technology, vol. 1, no. 1, pp. 1-13, 2012.

[38] X. Zhao, H. Zhou, Y. Tang, and H. Jia, "Travelling wave solutions for modified Zakharov-Kuznetsov equation," Applied Mathematics and Computation, vol. 181, no. 1, pp. 634-648, 2006.

[39] Z. B. Li and J. H. He, "Application of the fractional complex transform to fractional differential equations," Nonlinear Science Letters A, vol. 2, pp. 121-126, 2011.

[40] J. H. He, S. K. Elagan, and Z. B. Li, "Geometrical explanation of the fractional complex transform and derivative chain rule for fractional calculus," Physics Letters A, vol. 376, no. 4, pp. 257259, 2012.

[41] X. J. Yang, General Fractional Derivatives: Theory, Methods and Applications, CRC Press, New York, NY, USA, 2019.

[42] X. J. Yang, “A new fractal nonlinear Burgers' equation arising in the acoustic signals propagation," Mathematical Methods in the Applied Sciences, vol. 42, no. 18, pp. 7539-7544, 2019.

[43] X. J. Yang, Y. Y. Feng, C. Cattani, and M. Inc, "Fundamental solutions of anomalous diffusion equations with the decay exponential kernel," Mathematical Methods in the Applied Sciences, vol. 42, no. 11, pp. 4054-4060, 2019.

[44] X. J. Yang, "A new integral transform operator for solving the heat-diffusion problem," Applied Mathematics Letters, vol. 64, pp. 193-197, 2017.

[45] J. G. Liu, X. J. Yang, Y. Y. Feng, and M. Iqbal, “Group analysis to the time fractional nonlinear wave equation," International Journal of Mathematics, vol. 31, no. 4, article 2050029, 2020.

[46] J.-G. Liu, X.-J. Yang, Y.-Y. Feng, and H.-Y. Zhang, “Analysis of the time fractional nonlinear diffusion equation from diffusion process," Journal of Applied Analysis \& Computation, vol. 10, no. 3, pp. 1060-1072, 2020.

[47] J. G. Liu, X. J. Yang, and Y. Y. Feng, "On integrability of the time fractional nonlinear heat conduction equation," Journal of Geometry and Physics, vol. 144, pp. 190-198, 2019.

[48] W. Gao, P. Veeresha, D. G. Prakasha, and H. M. Baskonus, "New numerical simulation for fractional Benney-Lin equation arising in falling film problems using two novel techniques," Numerical Methods for Partial Differential Equations, vol. 5, 2020.

[49] M. Mohammad and C. Cattani, "Applications of bi-framelet systems for solving fractional order differential equations," Fractals, vol. 28, no. 8, article 2040051, 2020.

[50] J. Singh, D. Kumar, Z. Hammouch, and A. Atangana, “A fractional epidemiological model for computer viruses pertaining to a new fractional derivative," Applied Mathematics and Computation, vol. 316, pp. 504-515, 2018.

[51] E. F. D. Goufo, S. Kumar, and S. B. Mugisha, "Similarities in a fifth-order evolution equation with and with no singular kernel," Chaos, Solitons \& Fractals, vol. 130, article 109467, 2020.

[52] S. K. Panda, T. Abdeljawad, and C. Ravichandran, "A complex valued approach to the solutions of Riemann-Liouville integral, Atangana-Baleanu integral operator and non-linear telegraph equation via fixed point method," Chaos, Solitons \& Fractals, vol. 130, article 109439, 2020. 
[53] M. Mohammad and A. Trounev, "Implicit Riesz wavelets based-method for solving singular fractional integrodifferential equations with applications to hematopoietic stem cell modeling," Chaos, Solitons \& Fractals, vol. 138, article 109991, 2020.

[54] E. Ilhan and I. O. Kiymaz, "A generalization of truncated Mfractional derivative and applications to fractional differential equations," Applied Mathematics and Nonlinear Sciences, vol. 5, no. 1, pp. 171-188, 2020.

[55] H. Durur, E. Ilhan, and H. Bulut, "Novel complex wave solutions of the $(2+1)$-dimensional hyperbolic nonlinear Schrödinger equation," Fractal and Fractional, vol. 4, no. 3, p. 41, 2020.

[56] W. Gao, P. Veeresha, H. M. Baskonus, D. G. Prakasha, and P. Kumar, "A new study of unreported cases of 2019-nCOV epidemic outbreaks," Chaos, Solitons \& Fractals, vol. 138, article 109929, 2020.

[57] W. Gao, P. Veeresha, D. G. Prakasha, and H. M. Baskonus, "Novel dynamic structures of 2019-nCoV with nonlocal operator via powerful computational technique," Biology, vol. 9, no. 5, p. 107, 2020.

[58] W. Gao, H. M. Baskonus, and L. Shi, "New investigation of bats-hosts-reservoir-people coronavirus model and application to 2019-nCoV system," Advances in Difference Equations, vol. 2020, no. 391, 11 pages, 2020.

[59] W. Gao, G. Yel, H. M. Baskonus, and C. Cattani, "Complex solitons in the conformable $(2+1)$-dimensional AblowitzKaup-Newell-Segur equation," AIMS Mathematics, vol. 5, no. 1, pp. 507-521, 2020.

[60] A. Prakash, M. Goyal, H. M. Baskonus, and S. Gupta, “A reliable hybrid numerical method for a time dependent vibration model of arbitrary order," AIMS Mathematics, vol. 5, no. 1, pp. 507-521, 2020.

[61] A. Atangana and D. Baleanu, "New fractional derivatives with nonlocal and non-singular kernel, theory and application to heat transfer model," Thermal Science, vol. 20, no. 2, pp. 763-769, 2016.

[62] A. Atangana and I. Koca, "Chaos in a simple nonlinear system with Atangana-Baleanu derivatives with fractional order," Chaos, Solitons \& Fractals, vol. 89, pp. 447-454, 2016.

[63] K. M. Saad, A. Atangana, and D. Baleanu, "New fractional derivatives with non-singular kernel applied to the Burgers equation," Chaos, vol. 28, no. 6, article 063109, 2018.

[64] R. Sahadevan and T. Bakkyaraj, "Invariant analysis of time fractional generalized Burgers and Korteweg-de Vries equations," Journal of Mathematical Analysis and Applications, vol. 393, no. 2, pp. 341-347, 2012.

[65] C. Li, Q. Guo, and M. Zhao, "New solitary wave solutions of $(2+1)$-dimensional space-time fractional Burgers equation and Korteweg-de Vries equation," Computers \& Mathematcs with Applications, vol. 77, no. 8, pp. 2255-2262, 2019.

[66] A. Akbulut and F. Tascan, "Lie symmetries, symmetry reductions and conservation laws of time fractional modified Korteweg-de Vries (mkdv) equation," Chaos, Solitons \& Fractals, vol. 100, pp. 1-6, 2017. 